\section{Differential Infection of Mother and Runner Plant Generations by Verticillium dahliae in a High Elevation Strawberry Nursery}

\author{
Thomas R. Gordon ${ }^{1}$, Sharon C. Kirkpatrick ${ }^{1}$, Douglas V. Shaw ${ }^{2}$, and \\ Kirk D. Larson ${ }^{2}$ \\ University of California, Davis, CA 95616
}

Additional index words. chloropicrin, methyl bromide, vascular wilt, Fragaria $\times$ ananassa

\begin{abstract}
Strawberry cultivars Selva and Camarosa (Fragaria $\times$ ananassa Duch.) were grown at a high elevation nursery in soil that was either naturally infested with Verticillium dahliae or was rendered pathogen-free through preplant fumigation with 2 methyl bromide : 1 chloropicrin (wt/wt) at $392 \mathrm{~kg} \cdot \mathrm{ha}^{-1}$. Plants grown in fumigated soil were inoculated with a conidial suspension of $V$. dahliae, prior to establishment. Just prior to harvest, plants were rated for disease based on symptoms of Verticillium wilt. At the same time, petiole samples were taken from mother plants and each of three generations of runner plants, along with the stolons subtending each of the sampled runner plants. Petioles and stolons were cultured to assay for the presence of $\mathrm{V}$. dahliae, and scored as either infected or not infected. The experiment was conducted in each of two successive years, and the following conclusions were supported by results obtained in both years. First, symptoms of Verticillium wilt on mother plants of both cultivars were highly correlated with recovery of $\boldsymbol{V}$. dahliae from petioles, but runner plants were consistently free of symptoms even though they were often infected. Second, runner plants sustained lower infection frequencies than mother plants, with the differences being significant in most cases. Lastly, infection of runner plants was due, at least in part, to transfer of inoculum from infected mother plants; in some cases this appears to have been the exclusive mode of infection.
\end{abstract}

Verticillium wilt, caused by Verticillium dahliae, is a potentially devastating disease of strawberries (Fragaria $\times$ ananassa Duch.) (Paulus, 1990). In California, Verticillium wilt has been managed effectively through the use of preplant fumigation with methyl bromide and chloropicrin (Wilhelm and Koch, 1956). The regular use of an effective fumigant has probably rendered many fruit production fields free of the pathogen or nearly so. Disease problems can still occur, however, if transplants used to establish the fruit production fields are infected. This is typically prevented by preplant fumigation at the high elevation nurseries where strawberry plants are produced (Larson and Shaw, 2000). In contrast to fruit production areas, high elevation nurseries often grow strawberries in rotation with potatoes. Potatoes are highly susceptible to $V$. dahliae, and as a result soil inoculum densities of this fungus are often very high in potato production areas (Davis et al., 1996). Conse-

Received for publication 29 May 2001. Accepted for publication 8 Nov. 2001. We gratefully acknowledge Lassen Canyon Nursery for providing the land and labor needed to conduct the experiments on which this study is based, and research assistance from B. Aegerter, J. Beales, P. Bonello, M. Ostrowski, T. Takehara, and K. Wikler. This research was supported, in part, by grants from the California Strawberry Commission, and USDA Cooperative Agreement 58-5306-7-851.

${ }^{1}$ Plant Pathology Dept.

${ }^{2}$ Pomology Dept. quently, an insufficient fumigation can leave enough surviving propagules to allow for infection of the mother plants (i.e., the plants used to establish the nursery production field). With the impending loss of methyl bromide as a soil fumigant (Ristaino and Thomas, 1997), less than fully effective fumigations are likely

At high elevation strawberry nurseries, mother plants are established in the spring, and they produce stolons throughout the summer. The stolons form runner plants, which in turn produce additional stolons and runner plants. The runner plants are harvested in the autumn, and used to establish plantings for fruit production in the coastal growing districts of California. Typically, at least three generations of runner plants will reach commercial size during the course of a growing season (Larson, 1993).

If $V$. dahliae is in the soil, mother plants can become infected and may show symptoms of Verticillium wilt at some time during the growing season. If symptomatic plants are evident, runner plants harvested from that field may or may not be infected (Gordon and Shaw, unpublished observations). It is unclear whether or not the absence of symptoms on a runner plant is at all predictive of its infection status. The present study was undertaken to establish: 1) whether or not plants infected with $V$.dahliae could be identified based on symptoms; 2) to determine if infection frequency differs between successive generations of runner plants; to become more common. and 3) to assess the importance of inoculum transfer from mother plants to runner plants, via stolons. To extend the generality of our findings, data were collected for two different cultivars, in each of two successive years.

\section{Materials and Methods}

The studies described herein were conducted at a commercial strawberry nursery located near the town of Macdoel, in Siskiyou County, Calif. (lat. $41^{\circ} 8^{\prime} \mathrm{N}$, elev. $\approx 1300 \mathrm{~m}$ ). The experimental plantings were in fields in which potatoes had been grown in prior years; the most recent previous crop was alfalfa. Experiments were conducted in two successive years, 1997 and 1998.

In both years, soil samples were collected from each plot twice during the growing season. A composite sample was taken from each plot by removing 12 soil cores $(2 \mathrm{~cm}$ diameter), taken within the top $15 \mathrm{~cm}$ of the soil profile, in a random pattern and combining them into one sample. Thereafter, soil was well mixed and allowed to air-dry for about6 weeks at room temperature. Soil from each plot was assayed by dilution plating on pectate medium that was prepared as described by Huisman (1988), except that the $\mathrm{pH}$ was adjusted to 8.5 instead of 7.0. A total of five plates were inoculated for each soil sample, with the equivalent of $\approx 100 \mathrm{mg}$ of soil per plate. The assay was conducted a second time, 4-6 weeks later. The mean number of colonyforming units (CFUs) of $V$. dahliae per gram of soil was calculated based on the replicated assays of samples from each of two sampling dates.

In May 1997, plants were established in six different plots, two of which were fumigated with a combination of 2 methyl bromide : 1 chloropicrin (w/w) (MB : CP) at $392 \mathrm{~kg} \cdot \mathrm{ha}^{-1}$ prior to planting. These plots had no detectable inoculum of $V$. dahliae. Two other plots had been fumigated one year earlier (Spring 1996) with chloropicrin alone at $168 \mathrm{~kg} \cdot \mathrm{ha}^{-1}$, and were determined to have 0.5 and 3.0 CFUs of $V$.dahliae per gram of soil. The remaining two plots had never been fumigated and had 24 and 47.5 CFUs per gram of soil. The purpose of the treatments was to obtain a wide range of inoculum levels in the plots from which plants were to be sampled. Certified disease-free runner plants of the cultivars Camarosa and Selva were used to plant each of the six plots.

In both of the MB : CP fumigated plots, 10 plants of each cultivar were root-dip inoculated with an isolate of $V$. dahliae that was previously obtained from diseased strawberry plants at the location where the experiments were conducted. The inoculation was accomplished by immersing plant roots in an aqueous suspension of $10^{6}$ microconidia $/ \mathrm{mL}$. for 10 min immediately prior to planting. The two cultivars were planted in parallel rows within each plot, with $\approx 2 \mathrm{~m}$ separating the rows. In each of the other four plots, 20 plants of each cultivar were planted in a similar manner but were not inoculated.

During the growing season, runner plants were tagged to identify their generation as first 
(those directly connected via a stolon to a mother plant), second (connected to a first generation runner plant), or third (connected to a second generation plant). Within $3 \mathrm{~d}$ prior to the commercial harvest (1 Oct. for 'Camarosa' and 15 Oct. for 'Selva'), all plants were rated as either diseased or healthy based on visual symptoms of Verticillium wilt; symptoms included stunting, and wilting and die-back of leaves. To assay for infection by $V$. dahliae, a single petiole was removed from each mother plant in all six plots. In addition, petioles were taken from two first generation runner plants, two second generation runner plants, and one third generation runner plant associated with each mother plant. A segment, $2-5 \mathrm{~cm}$ in length, from the central region of each of the intervening stolons was collected as well. Thus, in most case, five petioles and five stolons were collected from the vegetative progeny of each mother plant. However, sampling was more limited where mother plants produced only one first and/or second generation runner plant, and/or no third generation runner plants. In total, isolations were attempted for 1019 tissue pieces (petioles and stolons) from 'Camarosa' and 1081 from 'Selva'.

All plant samples were returned to the laboratory and stored at $4{ }^{\circ} \mathrm{C}$ for up to $4 \mathrm{~d}$ prior to culturing from them. To determine whether or not $V$. dahliae was present, petioles and stolon segments were incubated on pectate medium for 2-3 weeks. Prior to their placement on the agar medium, tissue pieces were treated with a brief dip in $70 \%$ ethanol (to fully wet the surface), followed by $2 \mathrm{~min}$ in $1.0 \%$ sodium hypochlorite. Growth of $V$. dahliae was identified based on its growth from the ends of the tissue segment and the formation of characteristic microsclerotia within the agar medium (Huismann, 1988). Petiole and stolon segments were scored as either colonized or not, based on the presence or absence of $V$. dahliae in culture.

In 1998, a similar experiment was conducted in an adjacent field. In this case, experimental plantings were established in twelve separate plots, all of which had been part of a crop rotation experiment (Gordon et al., unpublished). Each of three plots received one of the four following treatments: 1) 2 years of a fall rye crop (Secale cereale L.), followed by fumigation with MB : CP at a rate of 392 $\mathrm{kg} \cdot \mathrm{ha}^{-1}$ in August 1997; 2) 2 years of a fall rye crop with no fumigation; 3 ) 2 years of a fall rye crop and a planting of rape (Brassica napus L.) in the spring of both years, and no fumigation; or 4 ) fallow for 2 years and no fumigation. As in 1997, the purpose was not to test for effects of the treatments themselves but rather to obtain a diverse sample of infected plants.

Separate soil samples were taken for each cultivar within a plot. This was accomplished by removing soil cores within $0.5 \mathrm{~m}$ of the row of the cultivar being sampled, rather than throughout the area in which the two cultivars were grown, as was done in 1997. Thus a mean inoculum density of $V$. dahliae was calculated for each cultivar in each set of three plots corresponding to different treatments. For 'Camarosa' and 'Selva', respectively, the mean inoculum densities for the nonfumigated plots were $27.0( \pm 5.0)$ and $24.0( \pm 5.5) \mathrm{CFUs} /$ gram for treatment 2 (rye only), $29.0( \pm 2.5)$ and $23.7( \pm 4.3) \mathrm{CFUs} /$ gram for treatment 3 (rye/mustard), and 10.7 ( \pm 1.9$)$ and $13.0( \pm 3.5)$ CFUs/gram for treatment 4 (fallow). Verticillium dahliae was undetectable in the plots fumigated with MB : CP (treatment 1).

As described for the 1997 experiment, parallel rows of the strawberry cultivars Camarosa and Selva were planted in each plot, and those planted in the fumigated plots were root dip inoculated prior to planting. In this case, each plot had ten plants of both cultivars. Tagging, rating, and sampling of plant material was conducted as described for the 1997 experiment with the following exceptions. Petioles (and corresponding stolons) were taken from three first and second generation runner plants, instead of two as in 1997; three samples also were taken of third generation plants, as opposed to one in 1997. These larger sample sizes reflected greater availability due to higher productivity in 1998 than in 1997. Also, in 1998, each petiole was stored separately with the stolon from which it originated, whereas in 1997, all petioles and stolons from the same generation were bulked as one sample for each mother plant. In total, isolations were attempted from 1710 tissue pieces from 'Camarosa' and 1867 from 'Selva' in 1998. Harvest dates were 2 Oct. and 15 Oct. for 'Camarosa' and 'Selva', respectively.

Because the experimental designs differed in the 2 years, data were analyzed separately. Mean infection proportions were calculated for each cultivar, for mother and runner plants of each generation, and for stolon segments of each generation. An arcsin square root transformation was used prior to conducting analysis of variance (ANOVA) (Sokal and Rohlf, 1995), to test for the significance of differences in infection proportion between replications, cultivars, treatments, and generations. ANOVAs were conducted using SAS procedure GLM (SAS Inst., 1988). Because transformations had little effect on the outcome of the statistical tests, results based on non-transformed data are presented here. Where treatment effects were significant, Tukey's allpairwise comparison test was used to determine which means were significantly different.

\section{Results}

In 1997, mother plants of both 'Camarosa' and 'Selva' in all six plots showed symptoms of Verticillium wilt, which included stunting and dieback. Some of the root-dip inoculated plants developed symptoms early in the season, and produced relatively few runner plants, whereas most of the mother plants grown in infested soil remained free of symptoms until just prior to harvest and were not obviously less productive than symptomless plants. There was a good correlation between visual symptoms of Verticillium wilt and recovery of $V$. dahliae from petioles. For 'Camarosa' and 'Selva', V. dahliae was isolated from petioles on $97 \%(71 / 73)$ and $95 \%(59 / 62)$, respec- tively, of mother plants rated as diseased based on visual symptoms. Verticillium dahliae was also isolated from petioles on $5.2 \%(2 / 38)$ of 'Camarosa' mother plants that were symptomless, but from none of the symptomless 'Selva' mother plants. Symptoms of Verticillium wilt were not evident on runner plants of either cultivar in any of the six plots.

Because the treatments (fumigated in 1996, fumigated in 1997, or not fumigated) corresponded to different inoculum levels (zero, low and high), they were kept separate by including treatment as a factor in a four-way ANOVA. The other three factors were replication, cultivar (Selva and Camarosa) and generation (corresponding to mother plants, three generations of runner plants, and three generations of stolons). This analysis revealed a significant treatment $\times$ cultivar interaction $(P<0.001)$, so data for the two cultivars were further analyzed separately.

Results of the ANOVAs for 1997 are shown in Table 1. For 'Camarosa', differences between replications were nonsignificant $(P=0.468)$, whereas the effects of treatment and generation were both significant, $P=0.002$ and $P=0.031$, respectively. The treatment $\times$ generation interaction was not significant, indicating that the ranking of the generations by infection frequency was not significantly altered by the presence of inoculum in the soil. The results for 'Selva' were essentially the same, except that the treatment $\times$ generation interaction was significant $(P=0.032)$ (Table 1$)$.

For 'Camarosa', infection frequency was highest in the mother plants and progressively lower in subsequent generations of plants and stolons (Fig. 1A), although differences between runner generations were not significant. In 'Selva', mothers plant had an infection frequency similar to that of first generation stolons, but significantly higher than in all subsequent generations (Fig. 1B).

Where mother plants were root-dip inoculated (treatment one), the overall mean infection rate was not significantly different between 'Selva' $(0.317 \pm 0.087)$ and 'Camarosa' $(0.254 \pm 0.0475)$. In naturally infested soil (treatments one and two), 'Camarosa' had a significantly higher infection rate than 'Selva' at both low and high inoculum densities; $0.446( \pm 0.076)$ vs. 0.123 $( \pm 0.027, P<0.001)$, and $0.635( \pm 0.066)$ vs. 0.394 ( $\pm 0.073, P=0.022)$, respectively.

As in 1997, mother plants in 1998 showed symptoms of Verticillium wilt in all plots, and this corresponded well with recovery of the pathogen from petioles; $V$. dahliae was recovered from $97.4 \%$ (75/77) and $95.9 \%$ (94/98) of the petioles sampled from symptomatic mother plants of 'Camarosa' and 'Selva', respectively. However, $V$. dahliae was also recovered from $17.4 \%(4 / 23)$ and $11.8 \%$ (2/17) of 'Camarosa' and 'Selva' mother plants that were symptomless. No symptomatic runner plants were observed in any of the plots for either of the two cultivars.

Differences in soil inoculum levels between plots receiving different treatments were significant $(P<0.001)$, so treatment was in- 
Table 1. Analysis of variance results for percent of plants (or stolons) infected with Verticillium dahliae at the end of the season in 1997 and 1998.

\begin{tabular}{|c|c|c|c|c|c|c|c|c|}
\hline \multirow[b]{3}{*}{ Source } & \multicolumn{4}{|c|}{1997} & \multicolumn{4}{|c|}{1998} \\
\hline & \multicolumn{2}{|c|}{ Camarosa } & \multicolumn{2}{|c|}{ Selva } & \multicolumn{2}{|c|}{ Camarosa } & \multicolumn{2}{|c|}{ Selva } \\
\hline & $\mathrm{df}$ & MS & df & MS & $\mathrm{df}$ & MS & df & MS \\
\hline Replication(R) & 1 & 0.0305 & 1 & 0.0260 & 2 & $0.1508^{* *}$ & 2 & $0.0761^{* *}$ \\
\hline Treatment $(\mathrm{T})$ & 2 & $0.5073^{* * *}$ & 2 & $0.2746^{* * *}$ & 3 & $0.1808^{* * *}$ & 3 & $0.0466^{*}$ \\
\hline Generation(G) & 6 & $0.1648^{*}$ & 6 & $0.3083^{* * *}$ & 6 & $0.3979^{* * * *}$ & 6 & $0.9205^{* * *}$ \\
\hline $\mathrm{T} \times \mathrm{G}$ & 12 & 0.0110 & 12 & $0.0310^{*}$ & 18 & 0.0164 & 18 & 0.0164 \\
\hline Error & 20 & 0.0555 & 20 & 0.0122 & 54 & 0.0247 & 54 & 0.0121 \\
\hline
\end{tabular}

*,**,***Significant at $P<0.05,0.01$, or 0.001 levels, respectively.

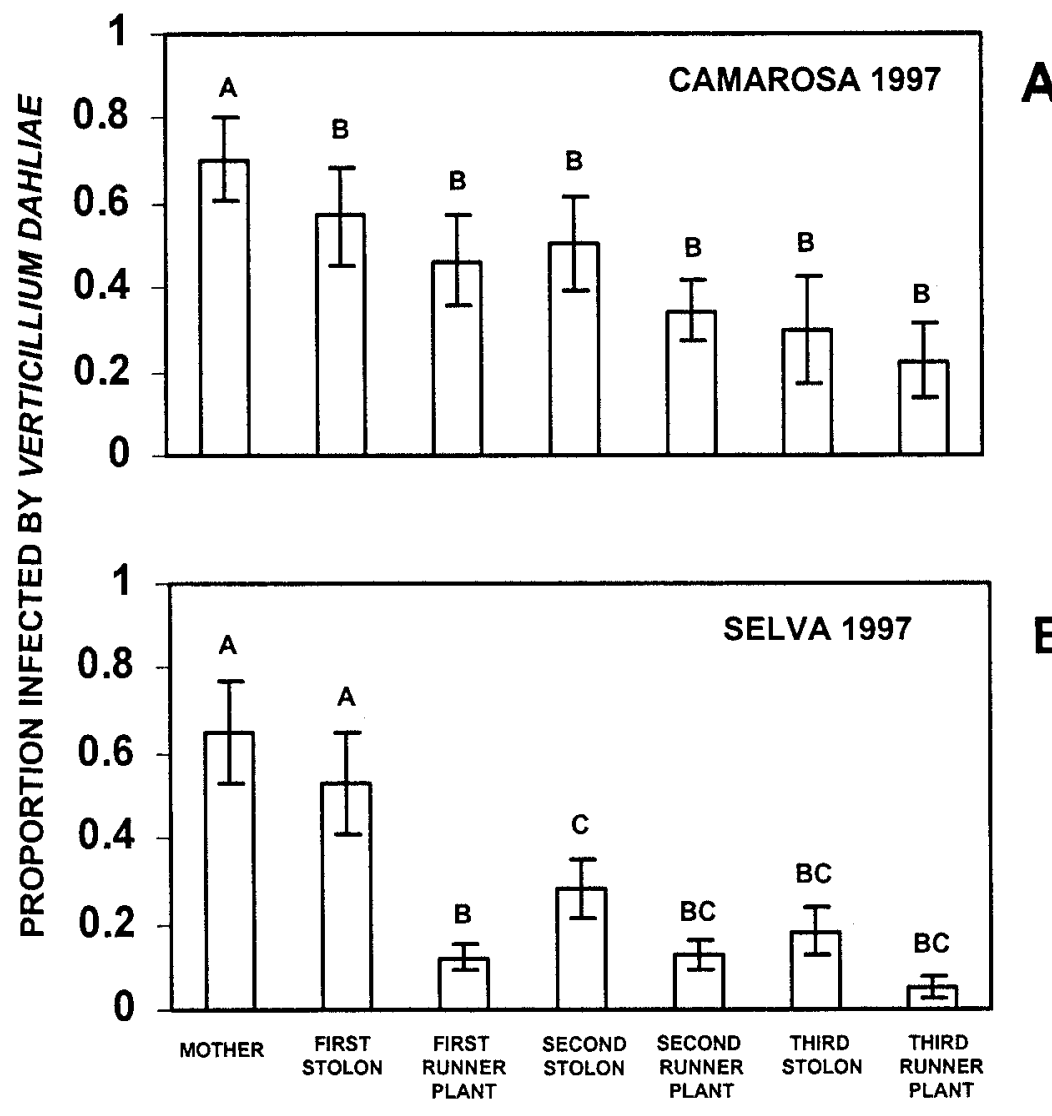

Fig. 1. (A) The height of each bar corresponds to the mean proportion of infected mother or daughter plants, or intervening stolon segments of the cultivar Camarosa in 1997. The length of the error bar corresponds to $2 \times$ the standard error (SE). Means denoted by a common letter were not significantly different according to Tukey's all pairwise comparison test $(P=0.05)$. The actual means \pm standard deviations (SD), and the number of observations ( $\mathrm{n}$ ) are as follows: mother plants, $0.703 \pm 0.239$ (97); first generation stolons, 0.570 \pm 0.283 (192); first generation runner plants, $0.464 \pm 0.260$ (194); second generation stolons, $0.505 \pm 0.280$ (182); second generation runner plants, $0.346 \pm 0.179$ (189); third generation stolons, $0.301 \pm 0.307$ (82); and third generation runner plants, $0.226 \pm 0.218$ (83). (B) The height of each bar corresponds to the mean proportion of infected mother or daughter plants, or intervening stolon segments of the cultivar Selva in 1997. The length of the error bar corresponds to $2 \times$ the standard error (SE). Means denoted by a common letter were not significantly different according to Tukey's all pairwise comparison test $(P=0.05)$. The actual means \pm SD, and the number of observations (n) are as follows: mother plants, $0.650 \pm 0.295$ (100); first generation stolons, $0.529 \pm 0.301$ (200); first generation runner plants, $0.121 \pm 0.071$ (200); second generation stolons, $0.283 \pm 0.163$ (197); second generation runner plants, $0.127 \pm 0.083$ (198); third generation stolons, $0.183 \pm 0.134$ (93); and third generation runner plants, $0.054 \pm 0.028(93)$

cluded as a factor in a four-way ANOVA. This analysis revealed significant interactions $(P<$ $0.001)$ between cultivar and generation, and between cultivar and treatment. Analyzing the data for the two cultivars separately indicated significant effects of replication, treatment, and generation for both 'Camarosa' and 'Selva' (Table 1). The treatment $\times$ generation interaction was not significant for either cultivar,
(Fig. 2B). Comparing cultivars within treatments, 'Camarosa' had a significantly higher infection frequency than 'Selva' for second generation runner plants, but otherwise differences were nonsignificant (data not shown). If data from runner plants were analyzed (threeway ANOVA) separately (i.e., stolons and mother plants were excluded), the infection frequency for 'Camarosa' was significantly higher than for 'Selva' $(P<0.001)$. In this case no interactions were significant.

For the samples taken in 1998, each petiole was stored separately with the stolon from which it originated (see materials and methods). Thus it could be determined that, for 'Camarosa', of 116 first generation runner plants that were infected, $91(78 \%)$ were subtended by an infected stolon; the remaining plants presumably sustained infections via their roots. For second and third generation runner plants, $85 \%$ and $98 \%$, respectively, of the infected plants were associated with an infected stolon. A similar trend was apparent in 'Selva': $87.9 \%$ (94/107), 88.9\% (32/36), and $95.2 \%(20 / 21)$ of infected plants were subtended by an infected stolon for first, second and third generations, respectively.

The results from both years suggest that the absence of inoculum in the soil does not affect the ranking of runner generations (stolons and plants) by infection frequency. This is indicated by the lack of a significant treatment $\times$ generation interaction in most cases (Table 1). The exception to this generality is 'Selva' in 1997, where this interaction effect was significant $(P=0.032)$. However, a combined analysis over studies (Fisher, 1932) demonstrated that treatment $\times$ generation interactions were nonsignificant when considered for all year and cultivar combinations $\left(\chi_{(8)}^{2}=10.59, P>0.23\right)$ or for Selva cultivar studies combined over both years $\left(\chi_{(4)}^{2}=7.70\right.$, $P>0.14)$. These combined results suggest that the single borderline significant treatment $\times$ generation interaction detected is likely the result of sampling error.

\section{Discussion}

The results from experiments conducted in two successive years support the following conclusions concerning Verticillium wilt in high elevation strawberry nurseries. First, whereas disease symptoms were strongly correlated with recovery of $V$. dahliae from mother plants (those used to establish nursery plantings), this was not the case with runner plants, which were frequently infected but always free of symptoms. Second, runner plants generally sustained lower infection rates than mother plants. Finally, the patterns of infection were consistent with runner plants receiving inoculum primarily from mother plants via stolons rather than from soilborne inoculum via their roots.

Previous work has shown that strawberry genotypes differ in their susceptibility to Verticillium wilt, based on root-dip inoculations of plants conditioned for fruit production (Shaw et al., 1997). Results from these studies indicated that 'Camarosa' was more suscep- 


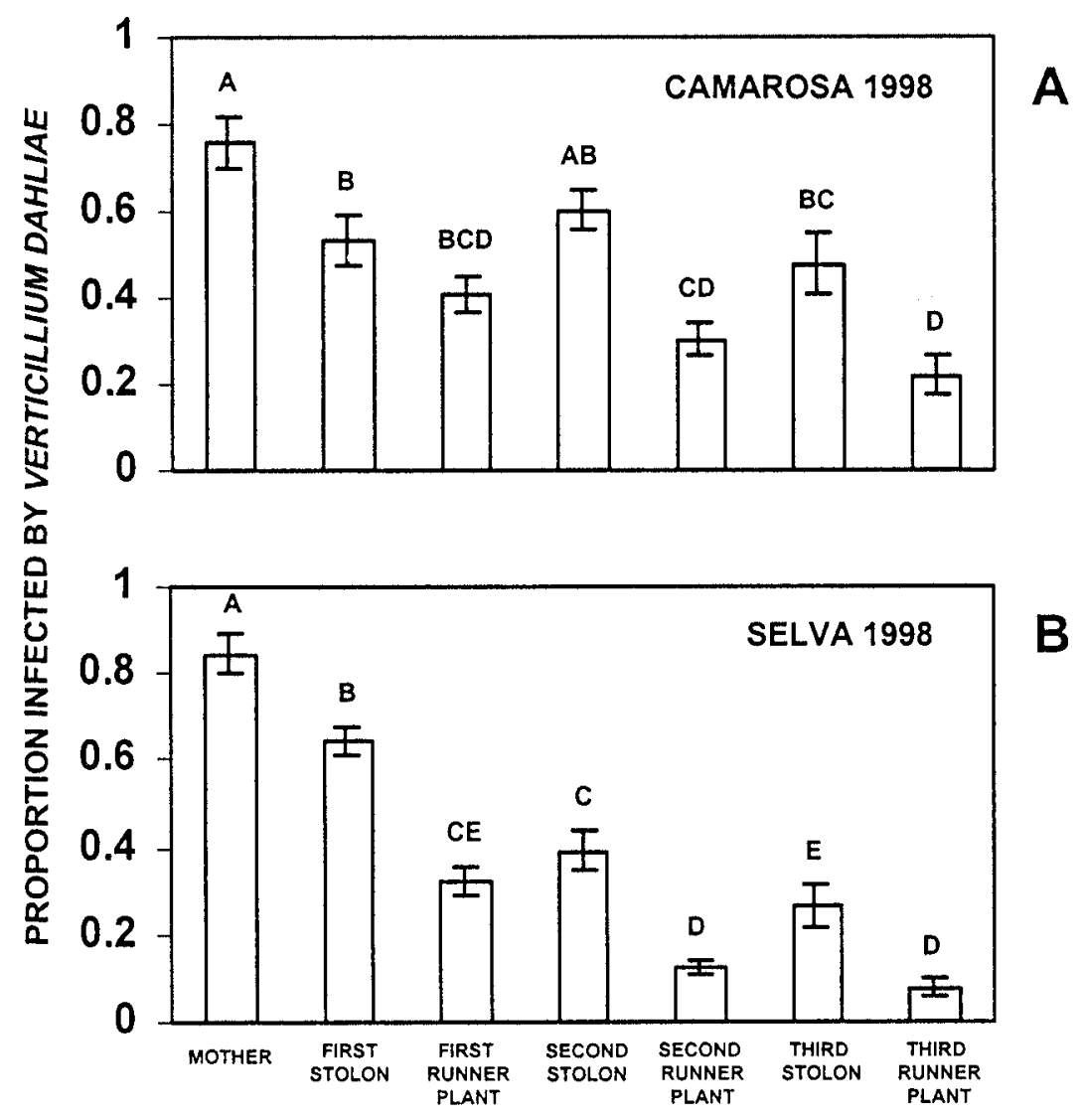

Fig. 2. A) The height of each bar corresponds to the mean proportion of infected mother or daughter plants, or intervening stolon segments of the cultivar Camarosa in 1998. The length of the error bar corresponds to $2 \times$ the standard error (SE). Means denoted by a common letter were not significantly different according to Tukey's all pairwise comparison test $(P=0.05)$. The actual means \pm standard deviations (SD), and the number of observations are as follows: mother plants, $0.756 \pm 0.203$ (103); first generation stolons, 0.534 \pm 0.193 (301); first generation runner plants, $0.409 \pm 0.157$ (289); second generation stolons, $0.603 \pm 0.165$ (271); second generation runner plants, $0.303 \pm 0.130$ (276); third generation stolons, $0.479 \pm 0.232$ (229); and third generation runner plants, $0.218 \pm 0.155(231)$. B) The height of each bar corresponds to the mean proportion of infected mother or daughter plants, or intervening stolon segments of the cultivar Selva in 1998. The length of the error bar corresponds to $2 \times(\mathrm{SE})$. Means denoted by a common letter were not significantly different according to Tukey's all pairwise comparison test $(P=0.05)$. The actual means \pm $\mathrm{SD}$, and the number of observations (n) are as follows: mother plants, $0.847 \pm 0.153$ (116); first generation stolons, $0.643 \pm 0.120$ (332); first generation runner plants, $0.323 \pm 0.105$ (332); second generation stolons, $0.390 \pm 0.161$ (297); second generation runner plants, $0.124 \pm 0.068$ (302); third generation stolons, 0.268 \pm 0.170 (244); and third generation runner plants, $0.077 \pm 0.066(244)$.

tible to Verticillium wilt, than 'Selva', although both were placed in an intermediate category (Shaw et al., 1997). In these tests, susceptibility was judged by the extent of symptom development on the inoculated plants. Under the conditions of our experiments, susceptibility could be gauged by this same criterion but also by the extent to which runner plants became infected. If only mother plants were compared, there was not a consistent difference between the two cultivars, but infection frequencies in runner plants tended to be lower for 'Selva' than for 'Camarosa'. Thus, the two cultivars may differ in the facility with which they transmit the pathogen from mother to runner plants.

Strawberry runner plant generations sustained lower rates of infection with $V$. dahliae than did mother plants. This difference is consistent with the fact that mother plants become rooted earlier than runner plants and thus presumably explore the soil more extensively, increasing the likelihood of encountering inoculum and sustaining infections. For the same reason, first generation runner plants would be expected to have a higher infection rate than subsequent generations. Although this was generally true, the differences were nonsignificant in most cases.

In addition to infection through their roots, runner plants may also receive inoculum directly from mother plants via stolons. Presumably, only the latter mechanism was operative where mother plants were root-dip inoculated, and the runner plants were growing in fumigated soil. Although one could postulate that soilborne infections resulted from inoculum densities below the threshold of detection $(0.5$ CFUs/g) (Harris and Yang, 1996), this seems unlikely given that noninoculated mother plants growing in fumigated soil nearby showed no symptoms of Verticillium wilt.
Infection of daughter plants may more commonly result from transmission of $V$. dahliae through stolons than from colonization of their roots. If this is so, the infection status of a stolon should be predictive of the infection status of the plant that develops from it. This appears to be the case, especially for third generation runner plants where greater than 95\% of infected daughters were subtended by an infected stolon, for both 'Camarosa' and 'Selva'. This fact coupled with a progressively shorter period of root growth and a correspondingly reduced opportunity for root infections, argues for the primary importance of stolon transmission in third generation runner plants.

Another way to evaluate the role of mother plants as a source of inoculum is to compare the infection frequencies of runner plants associated with infected and noninfected mother plants, where all the runner plants are growing in infested soil. For 'Selva', with data from both years combined, $97 \%$ of infected mother plants $(113 / 117)$ growing in infested soil were directly connected to at least one infected runner plant or stolon; only $6.1 \%$ of noninfected mother plants (3/49) growing under the same conditions were joined to at least one infected runner plant or stolon. For 'Camarosa', $97.5 \%$ of infected mother plants (116/119) formed infected runners or stolons, compared to $13.2 \%(5 / 38)$ of the noninfected mother plants. Thus, although the runner plants in question all had a comparable exposure to soilborne inoculum, those joined to an infected mother plant were clearly at higher risk of infection.

If an infection is established through vascular continuity between a mother and a runner plant, then the form of the pathogen and its localization in the host may be very different from what would occur if a colony in the root cortex gave rise to the systemic infection. In the former case, the fungus may exist only as spores in the xylem, and lack any presence in the root system. The significance of this distinction has not been established but it might help to explain why the viability of the pathogen in infected nursery plants is diminished by their exposure to low temperature after harvest (Shaw et al., 2002).

In summary, it is clear that in the absence of effective preplant fumigation infection by $V$. dahliae can pose a serious problem for production of disease-free nursery plants. The lack of symptoms on runner plants will require reliance on recognition of the disease in mother plants, testing for the presence of the pathogen in a subset of the plants destined for fruit production fields, or both.

\section{Literature Cited}

Davis, J.R., O.C. Huisman, D.T. Westermann, S.L. Hafez, D.O. Everson, L.H. Sorensen, and A.T. Schneider. 1996. Effects of green manures on Verticillium wilt of potato. Phytopathology 86:444-453.

Fisher, R.A. 1932. Statistical methods for research workers. 4th ed. Oliver and Boyd, London. 
Harris, D.C. and J.R. Yang. 1996. The relationship between the amount of Verticillium dahliae in soil and the incidence of strawberry wilt as a basis for disease risk prediction. Plant Pathol. 45:106-114.

Huisman, O.C. 1988. Seasonal colonization of roots of field-grown cotton by Verticillium dahliae and $V$. tricorpus. Phytopathology 78:708-716.

Larson, K.D. 1993. Nursery stolon series and rooting date, and subsequent plant growth and development in two strawberry cultivars. Amer. Soc. Hort. Sci. 1993 Annu. Mtg., Nashville,
Tenn., Prog. and Abstr. p. 99.

Larson, K.D. and D.V. Shaw. 2000. Soil fumigation and runner plant production: A synthesis of four years of strawberry nursery field trials. HortScience 35:642-646.

Paulus, A.L. 1990. Fungal diseases of strawberry. HortScience 25:885-889.

Ristaino, J.B. and W. Thomas. 1997. Agriculture, methyl bromide, and the ozone hole: Can we fill the gaps? Plant Dis. 81:964-977.

SAS Institute. 1988. SAS/STAT users guide, release 6.03 ed. SAS Inst., Cary, N.C.
Shaw, D.V., W.D. Gubler, and J. Hansen. 1997. Field resistance of California strawberries to Verticillium dahliae at three conidial concentrations. HortScience 32:711-713.

Shaw, D.V., T.R. Gordon, and K.D. Larson. 2002. Runner plant cold storage on reduces Verticillium dahliae Kleb. infection of nursery origin in strawberry. HortScience 37:932-935.

Sokal, R.R. and F.J. Rohlf. 1995. Biometry. 3rd ed. W.H. Freeman and Co.

Wilhelm, S. and E.C. Koch. 1956. Verticillium wilt controlled. Calif. Agr. 10:3-14. 